\title{
TEM STUDIES OF MICROSTRUCTURAL TRANSFORMATIONS IN THIN IRON FILMS INDUCED BY VACUUM ANNEALING
}

\author{
Enrico G. Keim* and Wojciech Lisowski** \\ ${ }^{*} \mathrm{MESA}^{+}$Research Institute, Central Materials Analysis Laboratory, University of Twente, P.O. Box \\ 217, 7500 AE Enschede, The Netherlands \\ ${ }^{* *}$ Institute of Physical Chemistry, Polish Academy of Sciences, Kasprzaka 44/52, PL-01-224 \\ Warszawa, Poland
}

Thin Fe films, evaporated on glass, appears to be a very useful catalytic material applied for "in situ" investigation of gas adsorption [1, 2] and multigas interactions [3, 4]. It is common practice for these films to be deposited at liquid nitrogen or room temperature, and then heated to higher reaction temperature. This annealing procedure changes the structure of the film. However, in spite of the wide applications, experimental investigation of Fe film structure during such annealing treatment is relatively scarce [5]. It is our aim to partially fill this gap by investigating the morphology and structure of thin Fe films evaporated onto a quartz substrate at $78 \mathrm{~K}$ followed by annealing within a wide temperature range. We have found that the temperature and duration of annealing play a crucial role in this process [6].

In this paper we present TEM results of a study dealing with the annealing-treatment induced transformation of approx. $50 \mathrm{~nm}$ thick Fe films on quartz at 700 and $1000 \mathrm{~K}$. The preparation of the iron films was performed in a glass UHV apparatus [7]. After evaporation the film samples were annealed "in situ", at a vacuum pressure lower than $10^{-6} \mathrm{~Pa}$, by heating the outer wall of the preparation quartz cell. The separated film samples were heated at 700 and $1000 \mathrm{~K}$ for 60 and 30 minutes, respectively. The annealing temperature was carefully monitored using two $\mathrm{Ni}-\mathrm{Cr}$ thermocouples fixed to the outer cell wall. Morphological and structural examination of the iron films was performed "ex situ" using TEM (Philips CM30 Twin (S)TEM). The TEM specimens were prepared in cross-section using dimpling and dual beam ion etching [8].

Vacuum annealing of Fe films evaporated at $78 \mathrm{~K}$ on a quartz substrate leads to their microstructural transformations: cross-section TEM analysis reveals a randomly oriented microcrystalline columnar structure of the Fe films annealed at $700 \mathrm{~K}$ (Fig. 1), the width of the crystalline columns being $30-100$ $\mathrm{nm}$. Electron microdiffraction reveals the existence of Fe grain families with different orientations, showing both low- and high- index Fe bcc diffraction patterns (representative diffraction patterns are presented in Fig. 1). Annealing performed at $1000 \mathrm{~K}$ causes the crystalline columns to become broader, reaching values of the order of $100-800 \mathrm{~nm}$, and is accompanied by a virtually complete reduction in the high-index Fe bcc diffraction patterns (Fig. 2). Well-oriented columnar structure in the (001) direction can be observed and mainly low-index planes were found in selected cross-section areas of the Fe film, indicating a true monocrystalline structure of well separated Fe grains (Fig. 2).

\section{References}

[1] G. Wedler et al., Surf. Sci. 47 (1975) 592.

[2] W. Lisowski, Polish J. Chem. 72 (1998) 756.

[3] G. Wedler and D. Borgmann, J. Catal. 44 (1976) 139.

[4] W. Lisowski, Appl. Surf. Sci. 147 (1999) 175.

[5] J.V. Sanders, in "Chemisorption and Reactions on Metallic films", Vol.1, Ed. J.R. Anderson, Academic Press, London (1971), 1. 
[6] W. Lisowski et al., Appl. Surf. Sci., in press.

[7] W. Lisowski, Vacuum 53 (1999) 13.

[8] E.G. Keim et al., J. Vac. Sci. Technol. A 19 (2001) 1191.

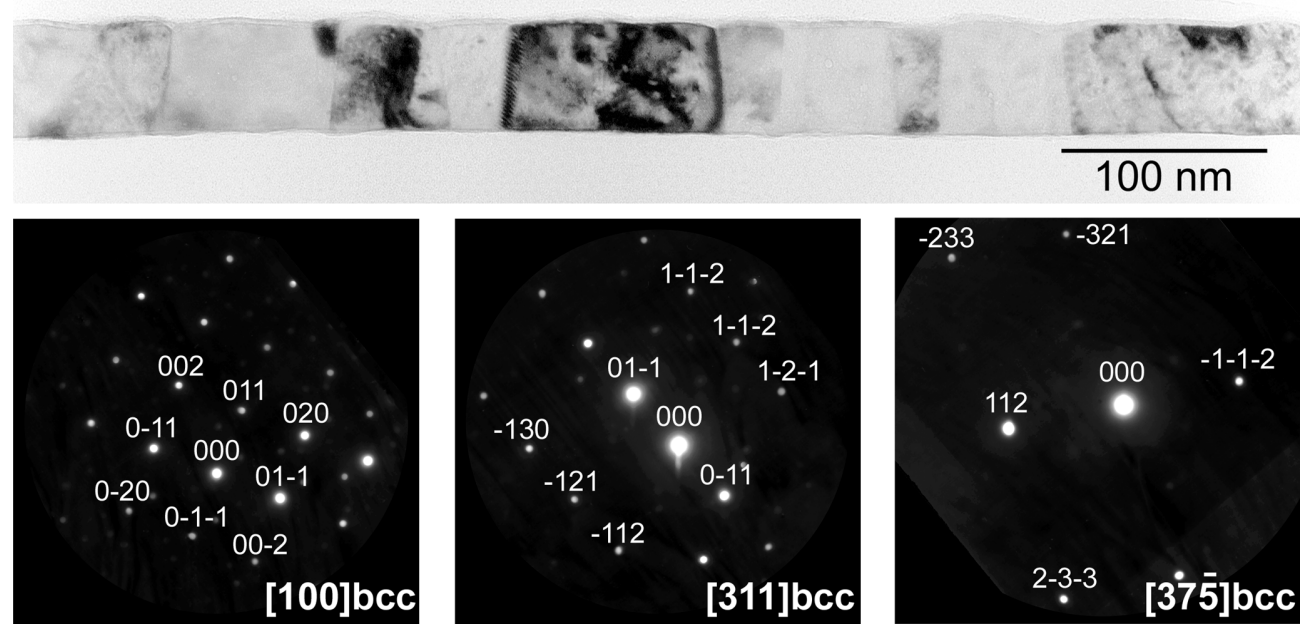

FIG. 1 Electron diffraction patterns of distinguishable areas in the cross-section of the Fe film (top image). The Fe film was annealed in vacuum at $700 \mathrm{~K}$ for 60 min. The thickness of the film is $54 \pm 2 \mathrm{~nm}$.

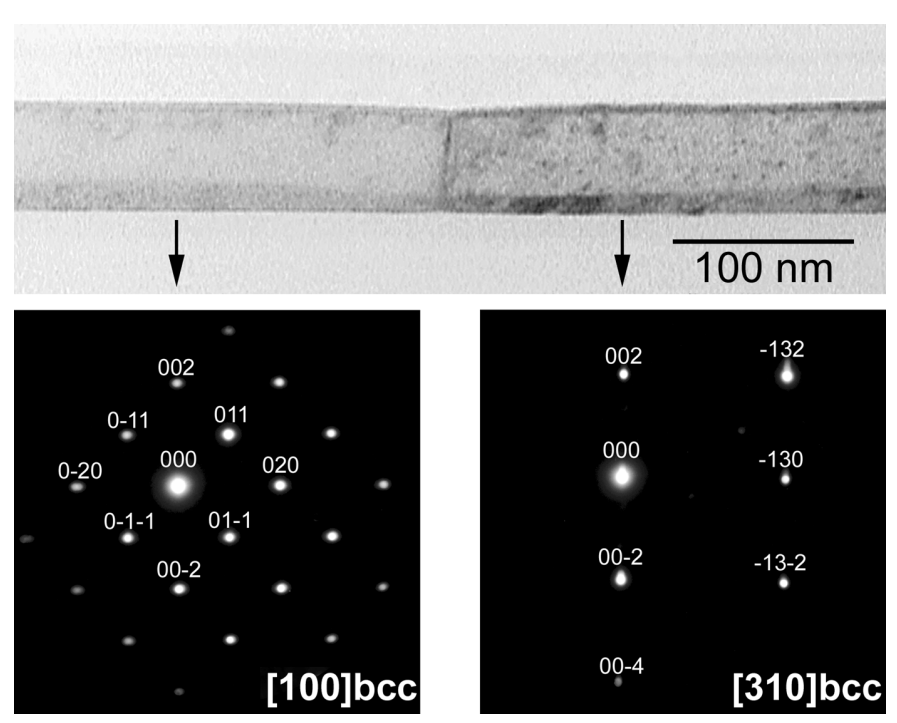

FIG. 2 Electron diffraction patterns of distinguishable areas (marked by arrows) in the cross-section of $\mathrm{Fe}$ film (top image). The Fe film was annealed in vacuum in two steps, at 700 and $1000 \mathrm{~K}$ for $60 \mathrm{~min}$ and $30 \mathrm{~min}$, respectively. The thickness of the film is $57 \pm 2 \mathrm{~nm}$. 